\title{
Constitutive modelling of gassy clay
}

\author{
Zhiwei $\mathrm{Gao}^{1, *}$ and $Y i \mathrm{Hong}^{2}$ \\ ${ }^{1}$ School of Engineering, University of Glasgow, G12 8QQ, UK \\ ${ }^{2}$ Key Laboratory of Offshore Geotechnics and Material of Zhejiang Province, College of Civil Engineering and Architecture, Zhejiang \\ University, Hangzhou, Zhejiang 310058, P.R. China
}

\begin{abstract}
Fine grained sediments with undissolved gas bubbles are widely distributed in the seabed around the world. The gas bubbles are much larger than the clay particles and fit in the saturated clay matrix rather than the pore water. Generally, these bubbles tend to degrade the soil stiffness and strength. But when the difference between the gas and pore water pressure is sufficiently small, pore water in the saturated clay matrix can drain into the cavities, making the void ratio of the saturated matrix smaller, which makes the undrained shear strength of the gassy clay sample higher than that of a saturated one. Such soil response cannot be described based on the assumption that gassy clay is a soil with compressible pore fluid. A new constitutive model for describing the stress-strain relation for gassy clay is proposed. An important feature of the model is that the gassy clay is considered as a composite material with compressible cavities which could be flooded by pore water. Effect of gas cavities on plastic hardening on the saturated matrix is accounted for. The model has been used to predict the response of three gassy clays and good agreement between the test data and model simulations is observed. Potential improvement of the model is discussed.
\end{abstract}

\section{Introduction}

Gas-charged marine sediments are widely distributed in the seabed throughout the world. For instance, gascharged seabed has been found in the Bristol Channel, North Sea, Gulf of Mexico, Gulf of Guinea and Eastern China Sea [1]. Gas can occur in the seabed in three ways: dissolved, undissolved in the form of gas-filled voids, or gas hydrates [2]. Dissolved gas has little effect on the physical properties of the seabed sediments unless the ambient pressure reduces, which creates gas bubbles in the soil [3]. Gas hydrates are only of concern for geotechnical engineering when they melt and release gas bubbles into the surrounding soil due to temperature or pressure change. But undissolved gas, mainly methane produced by decomposition of organic matter, thermal cracking of complex organic and inorganic compounds at great depth and gas exploration, is a major geohazard for offshore ground engineering, because it could dramatically change the mechanical response of soils, including stiffness, compressibility and shear strength [2].

The undissolved gas bubbles can have detrimental effect on offshore foundations in several ways [2]. In addition, they could also cause submarine landslides or sediment movement. In the deep sea with high pore pressure, these bubbles typically degrade the undrained shear strength of fine-grained soils. Submarine landslides in the gas-charged sediments can be triggered by a small earthquake, new sediment brought down by a flooding river or even a large storm [4]. There is concern that there could be more submarine landsides associated with gassy clays because global warming accelerates the decomposition of the organic materials in the soil [5].

Influence of undissolved gas on mechanical response of soil is deponent on the grain size. For fine-grained soils like clay, the gas bubbles are typically much bigger that the soil particles, and therefore, they fit in the soil matrix rather than the pore water [2]. This indicates that gassy clay is not a soil with compressible pore fluid. Instead, it is a composite material with saturated clay matrix and compressible cavities [2]. These cavities are similar to those in a solid like steel and concrete, which tend to degrade the material stiffness and strength. However, in some cases when the difference between the gas and pore water pressure is small enough, pore water in the saturated clay matrix can drain into these bubbles, which makes the void ratio of the saturated matrix smaller $[1,2]$. In a globally undrained test, the saturated clay matrix is essentially subjected to a partially drained loading condition when bubble flooding occurs. As a result, the undrained shear strength of the soil becomes higher $[1,2$, 3].

There have been extensive experimental studies on the compressibility and undrained shear strength of gassy clay but little progress has been made in constitutive modelling of this soil. The model proposed by Grozic et al. is based on the assumption that gassy clay is a soil with compressible fluid, which is not physically reasonable [6]. Consequently, it can only capture the beneficial effect of gas bubbles on the undrained shear strength of gassy clay, because compressible fluid makes the positive excess pore pressure smaller in an undrained test. The model proposed by Pietruszczak \& Pande can account for the

\footnotetext{
* Corresponding author: zhiwei.gao@glasgow.ac.uk
} 
case in which gas bubbles fit in the soil matrix. But it still fails to capture the detrimental effect of gas cavities on the undrained shear strength [7]. Thomas has proposed a technique for modelling consolidation of gassy clay, in which gas bubbles are considered as compressible solids [8]. This method gives good prediction for consolidation of gassy clay in oedometer tests but cannot describe the response of gassy clay in shear (like triaxial compression), in which the gas cavities can be flooded. Sultan \& Garziglia proposed a constitutive model which is specifically used for describing the structural damage caused by gas exsolution and expansion due to change in pore pressure [9].

A new approach for modelling the mechanical response of gassy clay will be presented in this paper, in which gassy clay is considered as a composite material with compressible cavities which can be flooded by pore water. The rest of the paper is organized as follows. First, the stress and strain variables and phase diagram of gassy clay are presented, which are essential for developing a constitutive model for gassy clay. A constitutive model in the triaxial stress space is then presented and verified using experimental data in the literature. Potential improvement for the model is also discussed.

\section{Stress and strain variables for gassy clay}

A composite approach should thus be used to model the stress-strain relation of gassy clay. According to the rule of mixture for composite materials [10], the total stress tensor $\sigma_{i j}$ for gassy clay can be decomposed as below

$$
\begin{gathered}
\sigma_{i j}=(1-f) \sigma_{i j}^{m}+f u_{g} \delta_{i j} \\
\sigma_{i j}^{m}=\sigma_{i j}^{\prime}+u_{w} \delta_{i j}
\end{gathered}
$$

where $f$ is the volume fraction of the gas cavities [2]. $\sigma_{i j}^{m}$ and $\sigma_{i j}^{\prime}$ are the total and effective stress tensor of the saturated clay matrix, $u_{g}$ is the gas pressure, $u_{w}$ is the pore water pressure and $\delta_{i j}$ is the Kronecor delta ( $=1$ for $i=j$ and $=0$ otherwise). The volume fraction of cavities $f$ is expressed as

$$
f=\frac{V_{c}}{V}
$$

where $V_{c}$ is the cavity volume and $V$ is the total soil volume. When the gas bubbles are not flooded, the volume of the gas is the same as that of the cavity [2],

$$
f=\frac{V_{c}}{V}=\frac{\left(1-S_{r}\right) e}{1+e}
$$

where $S_{r}$ is the degree of saturation and $e$ is the global void ratio. In most cases, $f$ is very small (less than 0.05 ). Therefore, contribution of the gas pressure to the total stress is negligible. In this paper, the following relation is assumed for the stress state for gassy clay by neglecting the effect of $f$ and $u_{g}$

$$
\sigma_{i j}=\sigma_{i j}^{\prime}+u_{w} \delta_{i j}
$$

The total strain tensor $\varepsilon_{i j}$ of a composite material can be decomposed as below using the rule of mixture

$$
\begin{gathered}
\varepsilon_{i j}=(1-f) \varepsilon_{i j}^{m}+f \varepsilon_{i j}^{c} \\
\varepsilon_{i j}=e_{i j}+\varepsilon_{v} \delta_{i j} \\
\varepsilon_{i j}^{m}=e_{i j}^{m}+\varepsilon_{v}^{m} \delta_{i j} \\
\varepsilon_{i j}^{c}=e_{i j}^{c}+\varepsilon_{v}^{c} \delta_{i j}
\end{gathered}
$$

where $e_{i j}$ is the shear strain tensor and $\varepsilon_{v}$ is the volumetric strain. The superscripts $m$ and $c$ denote saturated clay matrix and cavity, respectively. For the sake of simplicity, it is assumed that

$$
e_{i j}=e_{i j}^{m}=e_{i j}^{c}
$$

The total volumetric strain $\varepsilon_{v}$ is expressed as below based on Eqs. (7)-(8)

$$
\varepsilon_{v}=(1-f) \varepsilon_{v}^{m}+f \varepsilon_{v}^{c}
$$

Though $f$ is small, the term $f \varepsilon_{v}^{c}$ in Eq. (11) cannot be neglected, because $f \varepsilon_{v}^{c}$ can be much bigger than $(1-f) \varepsilon_{v}^{m}$ in some cases. The increment of $\varepsilon_{v}^{c}$ is related to the change of the cavity size

$$
d \varepsilon_{v}^{c}=\frac{d V_{c}}{V_{c}}
$$

where $d V_{c}$ denotes the volume change of the cavity.

The volumetric strain increment of the saturated clay matrix $d \varepsilon_{v}^{m}$ is dependent on the volume change due to water flow out/into the matrix at the boundary $d V_{b}$ bubble flooding $d V_{f}$ and compression of the pore water in the matrix $d V_{w}$

$$
d \varepsilon_{v}^{m}=\frac{d V_{b}+d V_{f}+d V_{w}}{V_{m}}=d \varepsilon_{v}^{b}+d \varepsilon_{v}^{f}+d \varepsilon_{v}^{w}
$$

where $d \varepsilon_{v}^{b}, d \varepsilon_{v}^{f}$ and $d \varepsilon_{v}^{w}$ denote the volumetric strain increments due to water flow at the boundary, bubble flooding and compression of pore water, respectively.

\section{A constitutive model for gassy clay in triaxial compression}

A constitutive model for gassy clay is presented in this section based on the framework above. The stress-strain relation of the saturated clay matrix is described based on 
the modified Cam-Clay model [11]. Volume change of gas bubbles is assumed to follow the Boyle's law. The cavities are assumed to have influence on plastic hardening of the saturated clay matrix. The material response is assumed to be rate-independent. The undissolved gas which forms the gas bubbles in the soil could become dissolved when there is increase in pore water pressure. On the other hand, the dissolved gas in the pore water can become undissolved as the pore water pressure decreases. For the sake of simplicity, such process is not accounted for in the present model.

\subsection{Constitutive model for the saturated clay matrix}

The yield function $F$ of modified Cam-Clay model is

$$
F=q^{2}-M^{2} p^{\prime}\left(p_{0}-p^{\prime}\right)=0
$$

where $p_{0}$ denotes the size of the current yield surface and $M$ is the critical state stress ratio in triaxial compression. The plastic flow rule is expressed as

$$
\begin{aligned}
& d \varepsilon_{v}^{m p}=\langle L\rangle \frac{\partial F}{\partial p^{\prime}} \\
& d \varepsilon_{q}^{m p}=\langle L\rangle \frac{\partial F}{\partial q}
\end{aligned}
$$

where $d \varepsilon_{v}^{m p}$ is the plastic volumetric strain increment, $d \varepsilon_{q}^{m p}$ is the plastic deviatoric strain increment, $L$ is the loading index and \langle\rangle are the McCauley brackets.

The elastic bulk modulus $K_{m}$ and shear modulus $G_{m}$ for the saturated matrix are

$$
\begin{gathered}
K_{m}=\frac{1+e_{m}}{\kappa} p^{\prime} \\
G_{m}=K_{m} \frac{3(1-2 v)}{2(1+v)}
\end{gathered}
$$

where $\kappa$ is the swelling index and $v$ is the Poisson's ratio which is assumed to be constant in the model.

The following hardening law is proposed based on the experimental observations

$$
d p_{0}=\langle L\rangle \frac{\partial F}{\partial p^{\prime}} \frac{\left(1+e_{m}\right) p_{0}}{\lambda-\kappa}\left[1-\frac{q \sqrt{f}}{M p^{\prime}}\left(\frac{u_{g}+p_{a}}{p_{0}}\right)^{\xi}\right]
$$

where $p_{a}$ is the atmospheric pressure $(101 \mathrm{kPa}), \lambda$ is the compression index and $\xi$ is a model parameter.

\subsection{Constitutive model for the saturated clay matrix}

It is assumed that the rate of bubble flooding related volume change $d \varepsilon_{v}^{f}$ can be expressed as $d \varepsilon_{v}^{f}=\left(1-S_{r}\right)\left(\frac{p_{0}}{u_{g}+p_{a}}\right)^{\xi} \exp \left(1-\frac{u_{g}-u_{w}}{x p_{0}}\right) \frac{\left\langle d u_{w}\right\rangle}{u_{g}+p_{a}}$

where $x$ is a positive model parameter. It is evident that $d \varepsilon_{v}^{f}=0$ when the bubble is completely flooded with $S_{r}=1$. The McCauley brackets are used to guarantee that bubble flooding occurs only when $u_{w}$ increases.

A simplified method for modelling the cavity volume change is used based on the Boyle's law for ideal gas

$$
\begin{gathered}
d \varepsilon_{v}^{c}=B d p=B\left(d p^{\prime}+d u_{m}\right) \\
B=\left\{\begin{array}{c}
0 \quad\left(S_{r}=1\right) \\
\frac{1}{u_{g}+p_{a}}\left(S_{r}<1\right)
\end{array}\right.
\end{gathered}
$$

\subsection{Initial gas pressure and gas pressure evolution}

The initial gas pressure before a test (either just before a consolidation test or just before shear after consolidation) is difficult to estimate, though there are upper and lower bounds for it [2]. In this paper, the initial gas pressure $u_{g i}$ is assumed to vary between the initial pore water pressure $u_{w i}$ and initial total stress $p_{i}$ as below

$$
u_{g i}=u_{w i}+\frac{u_{w}+p_{a}}{p_{i}+p_{a}}\left(p_{i}-u_{w i}\right)
$$

\section{Model simulation}

There are a total of 7 parameters for the model, 5 of which are identical with those in the Cam-Clay models $(M, \lambda$, $\kappa, v$ and $\mathrm{N}$ ). The parameter $x$ affects the rate of bubble flooding. Bigger $x$ indicates higher rate of bubble flooding. $\xi$ is used in both the plastic hardening law and bubble flooding. Bigger $\xi$ renders more reduction in the plastic modulus and bubble flooding, indicating more detrimental effect of bubbles on the stiffness and strength of gassy clay. It is recommend that $x$ should be determined to fit the stress-strain relation of gassy clay with high $f$ and low $u_{w}$ and $\xi$ can be determined to fit the stress-strain relation for tests with low $f$ and high $u_{w}$. The parameters for Malaysian kaolin with nitrogen are shown in Table 1.

Fig. 1 shows the model simulation for the response of Malaysian kaolin with nitrogen in isotropic compression [1].The matrix void ratio $e_{m}$ (or water void ratio) is calculated based on the water content and specific gravity of the soil particles. It is evident that there is a unique relation between $e_{m}$ and $p^{\prime}$, which is well captured by the model. This is because the effective stress principle works for the saturated clay matrix.

Fig. 2 shows the comparison between model simulations and test results on gassy Malaysian kaolin in undrained triaxial compression tests [1]. Generally, the model can capture both the beneficial and detrimental 
effect of gas bubbles on the undrained shear strength, while existing models can only capture the beneficial effect $[6,7]$. It gives good prediction for the undrained shear strength for all cases. But it overestimates the shear modulus before failure and fails to capture the effective stress path well for the tests on gassy samples with $u_{w i}>250 \mathrm{kPa}$. There are two possible reasons for this discrepancy. First, it could be due to that the yield surface used in the present model does not work for gassy clays with $u_{w i}>0$ (Fig. 2b). Yield surface with a flatter shape on the 'wet' side would give better simulations. Secondly, the elastic stiffness and plastic hardening law for the saturated clay matrix could be improved to get better model prediction. However, more parameters could be required for such improvement.

Table 1. Model parameters for Malaysian kaolin with nitrogen [1]

\begin{tabular}{|c|c|}
\hline Parameter & Value \\
\hline$M$ & 1 \\
\hline$\lambda$ & 0.0114 \\
\hline$\kappa$ & 0.035 \\
\hline $\mathrm{N}$ & 3.10 \\
\hline$\xi$ & 0.2 \\
\hline$x$ & 2.2 \\
\hline
\end{tabular}

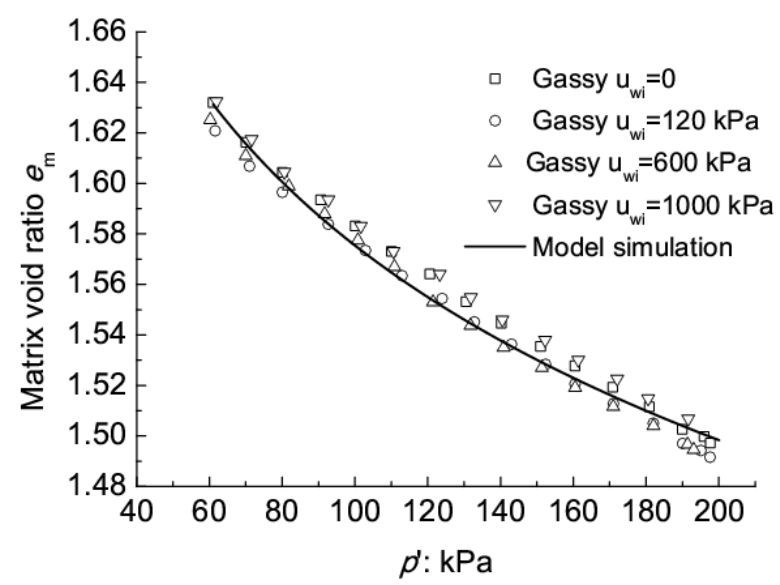

Fig. 1. Model simulation for Malaysian kaolin with nitrogen in isotropic compression.

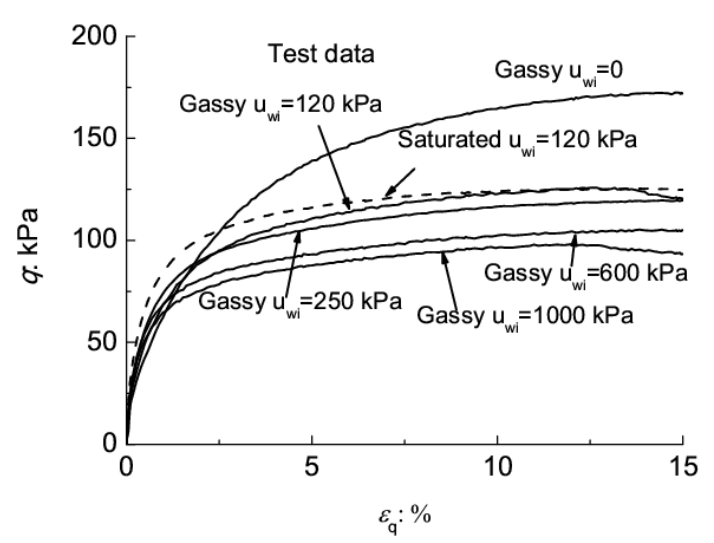

(a)

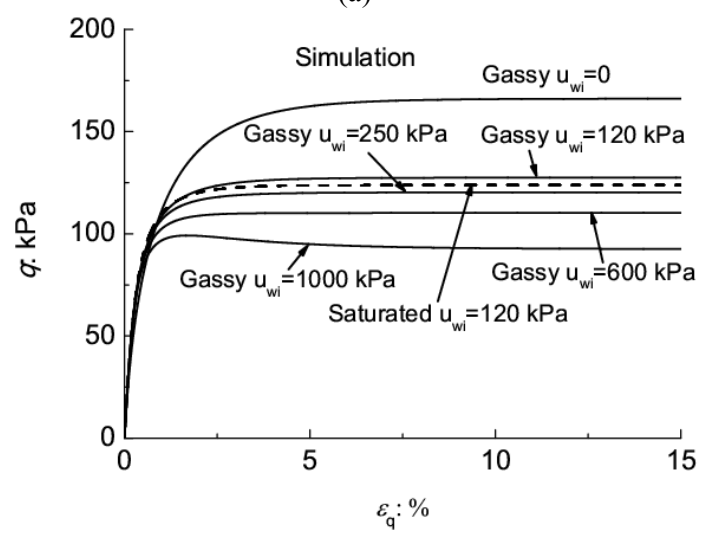

(b)

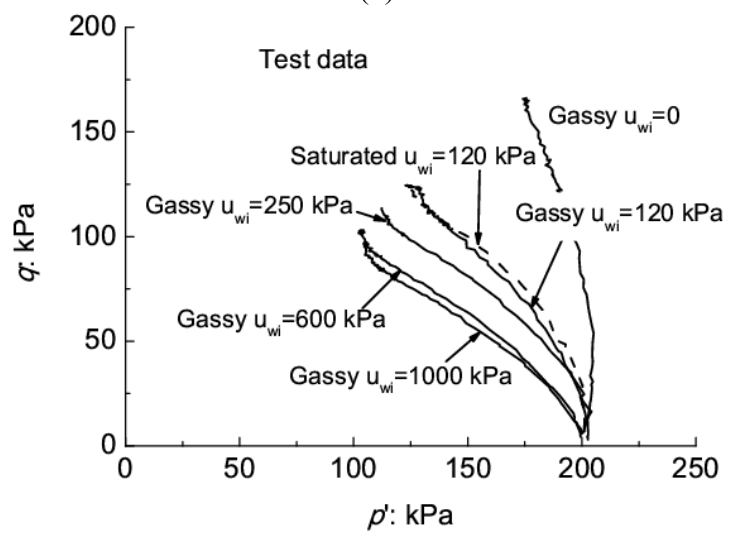

(c)

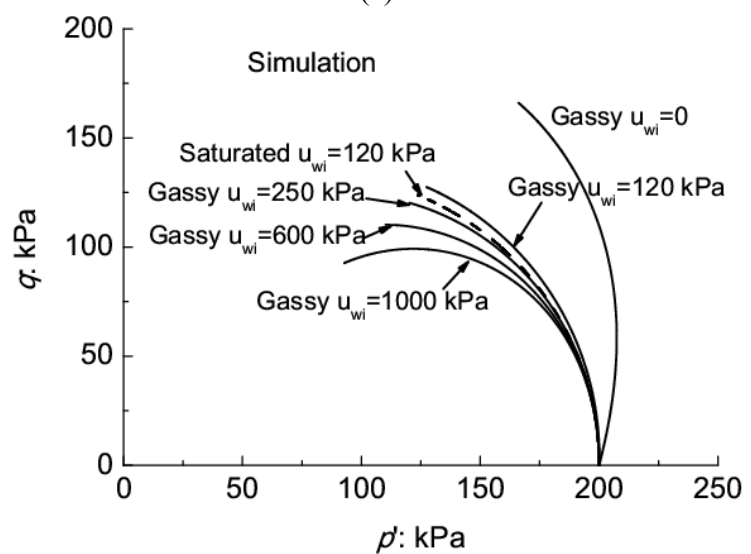

(d)

Fig. 2 Comparison between test data and model simulation for the behaviour of gassy Malaysian kaolin in undrained triaxial compression (Data from Hong et al., 2017) 


\section{Conclusion}

Undissolved gas bubbles can be found in both coarsegrained and fine-grained soils. For fine-grained soils like clay, these bubbles are much larger than the particles and fit in the soil matrix. Gassy clay must be considered as a composite material with compressible cavities, which could be flooded by pore water. A constitutive model for gassy clay is proposed based on this consideration. The gassy clay is considered as a composite material with compressible cavities. The stress and strain state of gassy clay is described using the rule of mixtures for composite materials. Compression of the cavities and bubble flooding occurs simultaneously. But the rate of bubble flooding is higher when the difference between gas pressure and water pressure is smaller. Effective stress principle is used to describe the stress-strain relation of the saturated clay matrix. As these cavities cause nonuniform distribution of strain and pore pressure in the matrix, which makes the matrix weaker. Therefore, the cavities are assumed to affect the plastic hardening of the matrix. This is important for modelling the detrimental effect of gas bubbles on the stiffness and strength of gassy clay. Compared to the modified Cam-Clay model, only 2 additional parameters are introduced to characterize the effect of gas bubbles on soil response. The model has been used to simulate the response of Malaysian kaolin with nitrogen. Good agreement between the model simulations and test data is observed. The model could be further improved by considering the effect of gas bubbles on elastic stiffness and yield surface shape. Better estimation of the initial gas pressure could also improve the model prediction.

The authors would like to acknowledge the fine contribution by Professor Simon Wheeler at University of Glasgow through various discussions. The second author is grateful for the financial supports from the National Natural Science Foundation of China (51779221) and Zhejiang Provincial Key Research and Development program (2018C03031).

\section{References}

1. Y. Hong, L. Wang, C.W. Ng, B. Yang, Can. Geotech. J., 54, 11 (2017)

2. S.J. Wheeler, PhD thesis, Oxford University, (1986)

3. N. Sultan, V. De Gennaro, A. Puech. Géotechnique, 62, 11 (2012)

4. J. Locat, H. J. Lee, Can. Geotech. J., 39, 1 (2002)

5. L. Milich, Global Env. Change, 9, 3 (1999)

6. J.L.H. Grozic, F. Nadim, T.J. Kvalstad, Comput. Geotech., 32, 7 (2005)

7. S. Pietruszczak, G.N. Pande, J. Geotech. Eng, 122, 1 (1996)

8. S. D. Thomas, PhD thesis, Oxford University, (1987)

9. N. Sultan, S. Garziglia, Géotechnique, 64, 11 (2014)

10. A. Diambra, E. Ibraim, D. Muid Wood, Geotext. and Geom., 28, 3 (2010)

11. D. Muir Wood, Critical state soil mechanics (1990) 